\title{
L'Atlas des Plécoptères de Suisse - Influence de la pollution
}

\author{
J. Aubert ${ }^{1}$
}

En Suisse, les cours d'eau de types variés du Jura, des Préalpes et des Alpes ont encore une faune plécoptérique relativement intacte. Par contre, ceux des régions agricoles et industrielles du Plateau ont perdu la moitié de leur faune dans leur cours moyen et même parfois la totalité dans leur cours inférieur.

L'atlas des Plécoptères donnera, par la méthode des quadrats, l'état actuel de nos connaissances sur la dist ribution géographique et sur l'écologie des espèces de Suisse.

The Stonefly Atlas of Switzerland, with notes on the influence of pollution (Plecoptera).

In Switzerland, many running water systems still retain nearly intact stonefly communities, particularly in the Jura, the Prealps and the Alps. However the watercourses in the industrial and agricultural regions of the Plateau have lost approximately half of their original species in their middle course and are often devoid of stoneflies in their lower reaches.

The Stonefly Atlas will provide, by means of a grid system, an overview of current knowledge on the geographical distribution and on the ecology of Swiss stoneflies.

J'ai commencé à étudier les Plécoptères en 1942. La thèse que j'ai publiée en 1946 donnait leur distribution géographique en Suisse romande, la description de quelques espèces nouvelles et des idées originales sur l'intérét que présentent les Plécoptères pour la zoogéographie.

De 1947 à 1949, j'ai étendu mes recherches à l'ensemble de la Suisse, puis jusque vers 1962, aux principaux massifs montagneux d'Europe occidentale et du bassin méditerranéen. Ensuite, et pendant une assez longue période, j'ai eu d'autres préoccupations scientifiques. Enfin, de 1978 à 1982, j'ai repris l'étude des Plécoptères de Suisse romande et des Préalpes de Suisse centrale.

De 1942 à 1949, je voyageais en train et terminais mes parcours en autobus, à pied ou à bicyclette. J'employais le simple filet entomologique et je ramassais les larves à la main, en tournant les pierres une à une. Depuis 1950, j'ai utilisé un véhicule à moteur, moto, puis auto et j'ai adopté une passoire

1. Musée zoologique, CH-1005 Lausanne, Suisse. métallique pour capturer les larves. Enfin, depuis 1978, j'ai récolté les adultes à l'aide d'un battoir. J'ai ainsi amélioré le rendement de mon travail sur le terrain et j'ai pu réunir près de trois fois plus d'insectes pendant ma seconde campagne de récoltes en Suisse.

Cette dernière campagne apporte deux espèces inédites pour le canton de Vaud, descomparaisons intéressantes sur l'évolution de la faune plécoptérique entre deux périodes espacées de trente ans. permettant de constater l'avance de la pollution des rivières et une documentation importante pour l'établissement d'un atlas des Plécoptères de la Suisse.

Leuctra pseudorosinae Aubert, 1954

J'ai décrit cette espèce d'après quelques exemplaires du versant nord des Préalpes autrichiennes. On la connaît aussi des Préalpes de Bavière. Je l'ai trouvée au col de Bretolet (Aubert 1965) à la frontière franco-suisse. Cette espece est commune dans un affluent de l'Orbe, le Biblanc, à la vallée de Joux. Je l'ai capturée à plusieurs reprises, au printemps, de 1978 à 1982 (10 o o , 15 \& \&). 
Nemoura sciurus Aubert, 1948

Cette espèce n'était connue de Suisse que par quelques exemplaires capturés à Lyss et dans les envi. rons de Bâle au début du siècle. $20 \sigma \%$ et $27 \%$ \& ont été récoltés dans deux stations du Jorat, près de Lausanne. Il s'agit de deux ruisselets, tributaires du Talent, qui coulent en forêt; l'un d'eux a un parcours rectiligne de deux à trois cents mètres entre deux champs cultivés, en dehors de la forêt. J'ai visité beaucoup de ruisselets forestiers dans d'autres régions du pays et je n'y ai jamais trouvé de Nemoura sciurus. Cette espèce est très printanière dans le Jorat où l'adulte disparaît avant la fin mai.

\section{Pollution et diminution de la faune}

Dans les Préalpes et les Alpes (cantons de Vaud, Fribourg, Lucerne et Unterwald), tous les divers types de cours d'eau sont riches en individus et en espèces ( 20 à 40 espèces par cours d'eau). Je n'ai pas observé de diminutions sensibles par rapport aux années 1942-1949. Il en est de même dans le Jura.

Sur le Plateau vaudois, qui atteint près de $1000 \mathrm{~m}$ d'altitude dans le Jorat, les rivières principales ont gardé la presque totalité de leur faune dans leur cours supérieur :

$$
1942-1949 \quad 1978-1982
$$

\begin{tabular}{|c|c|c|c|c|}
\hline Venoge & 29 & èces & 24 & pèces \\
\hline Aubonne & 26 & $"$ & 26 & $n$ \\
\hline Talent & 22 & $n$ & 26 & $"$ \\
\hline Broye & 21 & $"$ & 24 & $"$ \\
\hline
\end{tabular}

Deux de ces rivières (Talent et Broye) ont sans doute été mieux prospectées dans la seconde campagne.

Par contre, dans le cours inférieur des mêmes rivières, la situation est tout à fait différente :

\begin{tabular}{lrlrl} 
& \multicolumn{1}{c}{1942.1949} & \multicolumn{2}{c}{1978.1982} \\
Venoge, Préverenge & 15 espèces & \multicolumn{2}{c}{0 espèces } \\
$\quad$ Bussigny & 19 & $\prime$ & 3 & $"$ \\
Aubonne, Allaman & 12 & $\prime$ & 10 & $"$ \\
Talent, Chavornay & 7 & $\prime$ & 2 & $"$ \\
Broye, Payerne & 16 & $"$ & 9 & $"$ \\
Veveyse, Vevey & 24 & $\prime$ & 3 &
\end{tabular}

L'Aubonne à Allaman a été incomplètement prospectée dans deux campagnes. La Veveyse à Vevey. ajoutée ici, provient des Préalpes où sa faune est demeurée intacte.
Les espèces suivantes : Brachyptera trifasciata Pictet, B. monilicomis Pictet, Taeniopteryx schoenemundi Mertens, Dictyogenus imhoffi Pictet, Perla burmeisteriana Claassen ont disparu. Perlodes microcephalus Pictet est devenu très rare : il a disparu de la Venoge, du Talent et de la Broye. Je retrouve dans mes notes ce qui suit au sujet de cette espèce pour la Grande Emme à Burgdorf : a le 31 mars 1947, je trouvais des larves de P. microcephalus sous presque toutes les pierres, parfois même jusqu'à six ou sept sous un même caillou ". Je suis retourné à Burgdorf visiter la Grande Emme à trois reprises pour ne trouver qu'une seule larve le 21 mars 1983 ! Les grands Perlidae, sauf Perla grandis Rambur, qui est toujours commun dans les Préalpes et les Alpes, se sont passablement raréfiés. C'est apparemment la pollution industrielle qui est res. ponsable de cette situation dans le cours inférieur des rivières principales.

Les ruisseaux des environs de Lausanne ont subi, eux, les méfaits de l'urbanisation:

$\begin{array}{lcl} & 1942-1949 & 1978-1982 \\ \text { Flon } & 23 \text { espèces } & 0 \text { espèces } \\ \text { Paudèze et Chande- } & & \end{array}$

lard

27 " 7

Pour une série de 14 petits cours d'eau du Plateau (Promenthouse, Gèbre, Mèbre, Lutrive, Grenet, etc), la faune plécoptérique varie de cinq à neuf espèces par cours d'eau et le total n'est que de 18 espèces pour l'ensemble. Il s'agit de cours d'eau coulant dans des régions très cultivées et qui ont perdu plus de la moitié de leur faune plécoptérique en nombre d'espèces. Cette dégradation, due vraisemblable. ment à la pollution agricole, était manifeste déjà il $\mathrm{y}$ a trente ans. Les résultats des analyses chimiques n'ont pas encore été étudiés. On peut s'attendre à ce qu'ils viennent préciser la nature des différents polluants.

\section{Vers un atlas des Plécoptères de Suisse}

Par at las faunistique, on entend un ensemble de cartes de répartition géographique réalisées à l'aide de petits carrés ou quadrats de surfaces égales dans chacun desquels est notée la présence ou l'absence de l'espéce intéressée. Pour cela on constitue un fichier qui peut être traité par ordinateur. Un réseau de carrés de $10 \mathrm{~km}$ sur $10 \mathrm{~km}$ a été prévu pour l'Europe et plusieurs atlas ont déjà été publiés. 
Disposant déjà d'un fichier pour la répartition géographique des Plécoptères de Suisse, j'ai const $i$ tué, à partir de celui-ci, un autre par quadrats, puis réalisé des cartes pour chaque espèce. Ceci fait, on constate que, pour la fin de 1980 , les données pour l'ensemble des Plécoptères suisses manquent totalement pour 262 carrés (56\%); pour 107 carrés (23\%), on connaît entre 1 et 10 espèces et pour 99 carrés $(21 \%$ ) seulement, on a des connaissances relativement suffisantes (plus de 10 espèces). Toutefois, ces derniers carrés sont distribués d'une manière assez régulière sur la surface de la Suisse et les cartes montrent, avec assez de netteté, les principaux types de répartition : ainsi Perlodes jurassicus Aubert localisé au Jura, Capnioneura nemuroides Ris localisé aux Préalpes ou au contraire Nemoura cinerea Retzius commun dans toute la Suisse à toutes les altitudes.

Ces cartes ont surtout l'avantage de montrer les lacunes de nos connaissances et permettent de voir où il faut encore chercher des Plécoptères.

Pour les Plécoptères, qui ont été très étudiés au point de vue faunistique, on peut donc publier un atlas valable.

\section{Blbllographie}

Aubert (J.) 1946. - Les Plécoptères de la Suisse romande. Mitt schweiz ent. Ges., $20: 7-128$

Aubert (J.) 1949 a. - Plécopteres helvétiques. Notes morphologiques et systematiques. $\mathrm{lbid}, 22: 217-236$.

Aubert (J.) 1949 b. - Plècoptères helvétiques. Notes faunistiques et zoogéographiques. Bull. Soc. vaudoise sci. nat., 64 : $321-360$.

Auberı (J.). 1954. - Contribution à l'ètude du genre Leuct ro Stephens et description de quelques espèces nouvelles de ce genre. Mitt. schweiz. ent. Ges., 27 : 124-136.

Aubert (J.i. 1959. - Plecoptera, (Fauna) in Insecta Helvetica, vol. 1, $140 \mathrm{p}$.

Aubert (J.). - Les Plécoptères du canton de Vaud (à paraître). - Les Plécoptères du Napf et des Préalpes de Suisse cen. trale (à paraître).

- Plecoptera, (Catalogus) in Insecta Helvetica, sous forme d'un atlas (à paraitre). 